\title{
A near infrared view of Local Group dwarf galaxies
}

\author{
M. Gullieuszik ${ }^{1,2}$, E. V. Held ${ }^{1}$, Y. Momany ${ }^{2}$, \\ I. Saviane ${ }^{3}$, L. Rizzi ${ }^{4}$ and S. Ortolani ${ }^{2}$. \\ ${ }^{1}$ Osservatorio Astronomico di Padova, INAF, vicolo dell'Osservatorio 5, I-35122 Padova, Italy \\ ${ }^{2}$ Dip. di Astronomia, Università di Padova, vicolo dell'Osservatorio 2, I-35122 Padova, Italy \\ ${ }^{3}$ European Southern Observatory, Casilla 19001, Santiago 19, Chile \\ ${ }^{4}$ Institute for Astronomy, University of Hawaii, 2680 Woodlawn Drive, Honolulu, HI 96822
}

\begin{abstract}
We present the results of near-infrared imaging of the dwarf spheroidal galaxies Fornax and Leo I as part of a photometric survey of stellar populations in Local Group dwarf galaxies. Wide-field observations in the $J, H$, and $K_{s}$ bands have been obtained with the SOFI camera at the ESO NTT at La Silla, Chile. The aim of this project is to study the evolution of Local Group dwarf spheroidal galaxies, with special regard to star formation at intermediate epochs. The near-infrared data, together with optical catalogs from the Padova Local Group wide-field survey, provide a very large color baseline ideal to study the properties of red giant branch (RGB) and asymptotic giant branch (AGB) stars.
\end{abstract}

Keywords. Local Group, galaxies: dwarf, galaxies: individual (Fornax , Leo I), stars: AGB and post-AGB, infrared: galaxies.

\section{Near-infrared color-magnitude diagrams}

The infrared color-magnitude diagrams (CMD) of Fornax and Leo I are presented in Fig. 1 (upper panel). The CMDs show the extended AGBs of these two galaxies, characterized by prominent intermediate-age stellar populations, along with literature identification of carbon stars and other AGB stars. We have outlined some of the regions empirically defined by Nikolaev \& Weinberg (2000) to classify the evolved star content of the LMC from 2MASS photometry: upper RGB stars, oxygen-rich, carbon-rich, and dusty AGB stars. We note in particular the presence of dust-enshrouded AGB stars in both Leo I (see also Menzies et al. 2002) and Fornax.

\section{Distance and metallicity}

In the case of Fornax dSph, our photometry is deep enough to reach the "red clump" of core He burning stars above $1 M_{\odot}$. The $K_{s}$ magnitude of the red clump is one of the best distance indicators for resolved galaxies, since it is less affected from reddening and age dependence than optical bands. We adopted the Alves (2000) calibration and the prescriptions of Salaris \& Girardi (2002) to account for the actual star formation rate and chemical evolution. The resulting distance is $(m-M)_{0}=20.74$, in excellent agreement with the $I$-band RGB tip distance (Saviane et al. 2000).

The wide color baseline of optical-IR photometry is extremely useful to constrain the metallicity distribution function (MDF) of stars in galaxies. An MDF-related observable has been constructed by interpolating the location of red giant stars in the $M_{K_{s}},\left(V-K_{s}\right)$ CMD against the fiducial RGB lines of Galactic globular clusters (Valenti et al. 2004). 

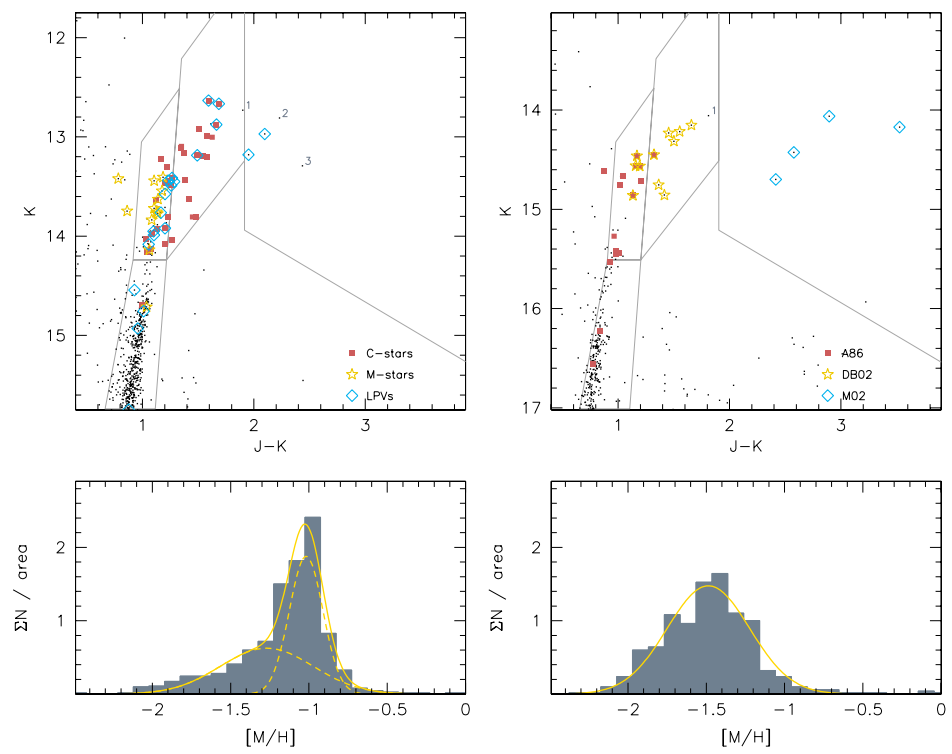

Figure 1. Upper Left: The near-IR CMD of Fornax along with literature classification of C-stars, M-giants, and Long Period variable stars (Stetson et al. 1998 and refs. therein; Bersier \& Wood 2002, Westerlund et al. 1991). Upper Right: The CMD of Leo I dwarf spheroidal. C-stars were identified by Azzopardi et al. (1986), Demers \& Battinelli (2002), and Menzies et al. (2002) using spectroscopy (A86), narrow-band (DB02) or infrared (M02) photometry. Lower Left: The observed MDF of Fornax. Lower Right: The observed MDF of Leo I.

The MDFs of Fornax and LeoI are shown in Fig. 1 (lower panels). This distribution exactly represents the distribution of stellar metallicities only in the case of old stellar populations. In the general case of extended star formation and younger stellar populations, as in Fornax and LeoI, these observed distribution functions still represent an important observable for the chemical evolution models to confront with. The observational MDF derived for Fornax is well modeled by the sum of two Gaussian functions. The principal component is centered at $[\mathrm{M} / \mathrm{H}]=-1.02$, with a tail of metal-poor stars. The MDF of Leo I is more like a single wide Gaussian centered at $[\mathrm{M} / \mathrm{H}]=-1.56$. Using theoretical isochrones to estimate the effects of age and metallicity on $\left(V-K_{s}\right)$ colors, we obtain $[\mathrm{M} / \mathrm{H}] \sim-0.9$ and $[\mathrm{M} / \mathrm{H}] \sim-1.2$ as our preliminary estimates of the age-corrected mean metallicities of Fornax and Leo I.

\section{References}

Alves, D.R. 2000, ApJ 539, 732

Azzopardi, M., Lequeux, J. \& Westerlund, B.E. 1986, A\& $A$ 161, 232

Bersier, D. \& Wood, P.R. 2002, AJ 123, 840

Demers, S. \& Battinelli, P. 2002, AJ 123, 238

Menzies, J., Feast, M., Tanabé, T., Whitelock, P. \& Nakada, Y. 2002, MNRAS 335, 923

Nikolaev, S. \& Weinberg, M.D. 2000, ApJ 542, 804

Salaris, M. \& Girardi, L. 2002, MNRAS 337, 332

Saviane, I., Held, E.V. \& Bertelli, G. 2000, ApJ 355, 56

Stetson, P.B., Hesser, J.E. \& Smecker-Hane, T.A. 1998, PASP 110, 533

Valenti, E., Ferraro, F.R. \& Origlia, L. 2004, MNRAS 351, 1204

Westerlund, B.E., Edvardsson, B. \& Lundgren, K. 1987, A\&\&A 178, 41 